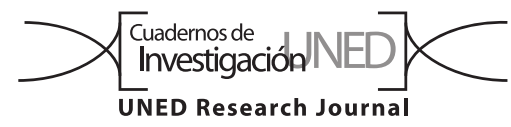

\title{
La plaga de langostas Schistocerca sp. (Orthoptera: Acridae) y su relación con el Mega Niño de 1877-1878 en Costa Rica
}

\author{
Ronald Eduardo Díaz Bolaños ${ }^{1}$, Eric J. Alfaro Martínez² \& Leninger Leitón Gutiérrez ${ }^{3}$ \\ 1. Universidad Estatal a Distancia (UNED), Escuela de Ciencias Sociales y Humanidades, 2050 San José, Costa Rica; Universidad de Costa Rica \\ (UCR), Centro de Investigaciones Geofísicas y Escuela de Estudios Generales, 2060 San José, Costa Rica; ronald.diaz@ucr.ac.cr \\ 2. Universidad de Costa Rica (UCR), Centro de Investigaciones Geofísicas, Centro de Investigación en Ciencias del Mar y Limnología y Escuela de \\ Física, 2060 San José, Costa Rica; erick.alfaro@ucr.ac.cr \\ 3. Universidad de Costa Rica (UCR), Escuela de Estadística y Escuela de Salud Pública, 2060 San José, Costa Rica; leninger.leiton@ucr.ac.cr
}

Recibido 08-VI-2018 • Corregido 14-IX-2018 • Aceptado 22-X-2018

\begin{abstract}
The locust Schistocerca sp. (Orthoptera: Acridae) plague and its relationship with the Mega Niño of 1877-1878 in Costa Rica". Introduction: locust plagues have been recorded for thousands of years and were feared by many societies. Objective: to assess if the locusts that affected Costa Rica in the 19th century were related with the Mega-Niño of 1877-1878. Methods: we checked files in the Costa Rican Congress, Governance, Municipalities and Police Force. Results: clouds of locusts were reported from Guanacaste in June 1876 and in the following months the reports spread to a large part of the country, with a lesser extent in Limón. The plague affected mainly the Central American Dry Corridor (CADC) while the Mega-Niño was developing. Conclusion: meteorological data support the hypothesis that conditions generated by the Mega-Niño favored the propagation of locusts that significant damaged agriculture. The pest was combated by the army and, by the church, with prayers, until it moved to Colombia.
\end{abstract}

Keywords: Costa Rica, history, climate, El Niño, environment, agriculture, locusts.
RESUMEN: Introducción: las plagas de langostas se han registrado durante miles de años y fueron temidas por muchas sociedades. Objetivo: evaluar si las langostas que afectaron a Costa Rica en el siglo XIX estaban relacionadas con el Mega Niño de 1877-1878. Métodos: verificamos los archivos en el Congreso, Gobierno, Municipios y Policía de Costa Rica. Resultados: Guanacaste reportó nubes de langostas en junio de 1876 y en los meses siguientes los informes se extendieron a gran parte del país (en menor medida en Limón). La plaga afectó principalmente al Corredor Seco Centroamericano (CSC) cuando el MegaNiño se estaba desarrollando. Conclusión: los datos meteorológicos apoyan la hipótesis de que las condiciones generadas por el Mega Niño favorecieron la propagación de langostas que dañaron significativamente la agricultura. La plaga fue combatida por el ejército y, con oraciones, por la iglesia, hasta que se trasladó a Colombia.

Palabras claves: Costa Rica, historia, clima, El Niño, medio ambiente, agricultura, langostas.
En el período 1877-1878, el planeta Tierra se vio afectado por la presencia de un Mega-Niño, es decir, un evento característico del fenómeno de El Niño de inusual magnitud, en donde la interacción oceánica y atmosférica que se presenta a partir del calentamiento de las aguas del Océano Pacífico oriental frente a las costas sudamericanas, produce cambios en el comportamiento de las corrientes marinas asociados a impactos muy fuertes en el clima a escala global (Mörner, 1993; Alfaro \& Amador, 1996; Díaz \& Alfaro, 2016). De acuerdo con Aceituno et al. (2009), éste fenómeno se caracterizó por intensas sequías en el altiplano andino, el Nordeste de Brasil, África, India, Sudeste Asiático y el norte de China y abundantes lluvias e inundaciones en la cuenca del río Paraná, el centro de Chile, norte de Perú y el sur de Ecuador, impactos que provocaron millones de muertes en los territorios afectados y por eso se le considera uno de los más fuertes que se presentó en el siglo XIX.

En Costa Rica, la presencia del Mega-Niño coincide con la propagación de una plaga de langostas que se va a extender por la mayor parte del país (Díaz \& Alfaro, 2016). Estos insectos pertenecen a la especie conocida como langosta centroamericana (Schistocerca piceifrons piceifrons Walker, 1870), que habita desde México hasta Costa Rica y ha causado devastación en las tierras agrícolas desde épocas antiguas. Se conservan fuentes documentales que indican su presencia en América Central durante el período colonial y para el caso Costa Rica se han reportado nubes de langostas en los años 1659, 
$1665-1666,1688,1730,1771,1774,1798-1800$, las cuales provocaron daños en cultivos y sementeras, además de los reportes posteriores documentados para la década de 1850 y en el bienio 1877-1878 (Payne, 1988; Amador \& Muñoz, 2015).

Este insecto se ha caracterizado por desplazarse a través del istmo centroamericano en bandadas, por lo que su radio de acción puede abarcar centenares de kilómetros. Peraldo, Solano y Quesada (2011) citando a Hilje et al. (2008) sostienen que su área de crianza se ubica en el territorio hondureño, propiamente en el Golfo de Fonseca (vertiente pacífica) y en el Valle del río Aguán (vertiente caribeña), desde donde se desplaza hacia otras zonas del istmo, por lo que suele penetrar en Costa Rica una vez que las nubes de langostas han recorrido territorio nicaragüense.

Según el Ministerio de Agricultura y Ganadería de Costa Rica (2015) en el documento Guía técnica para el muestreo de la langosta voladora, se establece una ficha técnica que permite la caracterización de este insecto (Cuadro 1).

En relación con lo anterior, SENASICA-DGSV (2016) incluye la siguiente información presentada en una ficha técnica sobre la langosta centroamericana y su incidencia en México. Esto amplía la caracterización taxonómica de la langosta al incluir su sinonimia.

$\begin{array}{ll}\text { Sinonimia } & \text { Schistocerca americana americana } \\ & \text { (Astacio, 1981) } \\ & \text { Schistocerca vicaria (Astacio, 1981) }\end{array}$

Nombre común Langosta centroamericana (español) Central American locust (inglés)
CUADRO 1

Ficha técnica de la "langosta voladora"

\begin{tabular}{cl} 
Nombre Científico & \multicolumn{1}{c}{ Schistocerca piceifrons piceifrons. } \\
Nombre común & $\begin{array}{l}\text { Langosta voladora, chapulín, } \\
\text { saltamontes. }\end{array}$ \\
Posición taxonómica & Reino: Animal \\
& Phylum Arthropoda \\
& Clase: Hexapoda (Insecta) \\
& Subclase: Pterigota \\
& Orden: Orthoptera \\
& Sub Orden: Caelifera \\
& Super familia: Acridoidea \\
& Familia: Acrididae \\
& Sub-familia: Cyrtacanthacridiane \\
& Género: Schistocerca \\
& Especies: piceifrons, nitens, pallens \\
\hline
\end{tabular}

Fuente: SENASICA-DGSV (2016).

Tal y como se muestra en la Fig. 1, la langosta voladora presenta diferentes estadios biológicos que van desde los huevos hasta su forma adulta. En este sentido cabe destacar que, de acuerdo con las fuentes históricas consultadas, los nombres que mayormente se le daba al insecto era saltón y langosta lo que está en relación con las etapas de ninfas y adultas (voladoras) respectivamente, por lo que los observadores de la época pudieron identificar el estadio en que se encontraba el insecto una vez que se instalaba en una zona y así lo consignaban en los documentos. En otras fuentes, se le denomina chapulín, nombre con que habitualmente se le conoce en tierras mesoamericanas $y$, por tanto, así lo consignaron quienes observaban el avance de la plaga por territorio costarricense.

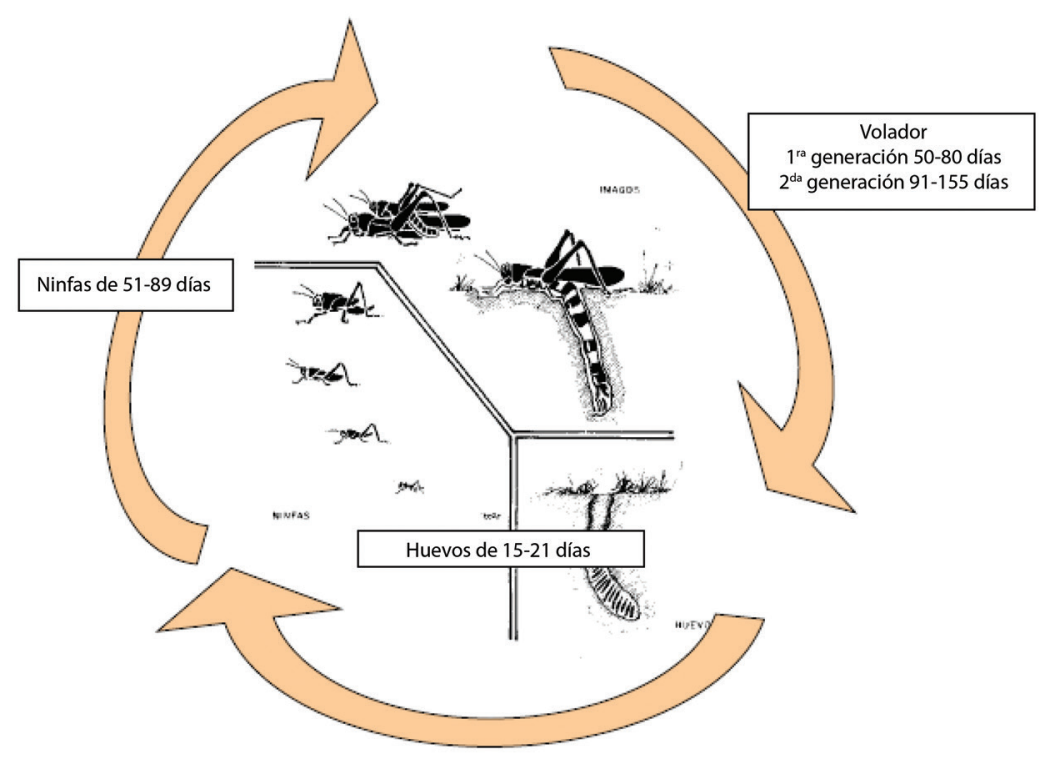

Fig. 1. Estados biológicos de la langosta voladora Schistocerca piceifrons piceifrons según Ministerio de Agricultura y Ganadería (2015). 
Aunado a lo anterior este ciclo biológico consta de dos estados extremos: 1) la fase solitaria que se caracteriza porque los insectos se encuentran dispersos y viven como saltamontes comunes y 2) la fase gregaria donde estos pueden formar concentraciones de hasta ochenta millones de insectos por $\mathrm{km}^{2}$ y consumir alrededor de cien mil toneladas de alimento verde por día (Retana, 2000; Flores, 2015). Por tanto, se denomina gregarización al mecanismo por medio del cual los insectos cambian de fase, normalmente condiciones ambientales que favorecen su reproducción masiva y les permite conformar nubes de langostas integradas por millones de insectos (Retana, 2000).

El objetivo de la presente investigación es analizar la relación existente entre la plaga de langostas en Costa Rica y el Mega-Niño que se presentó en el período 18771878, debido a que, de acuerdo con la información meteorológica disponible, las condiciones meteorológicas favorecieron la multiplicación de las langostas e hicieron posible su extensión por distintas zonas del país.

\section{MATERIALES Y MÉTODOS}

La metodología consiste en una revisión bibliográfica basada en la consulta de fuentes documentales primarias conservadas en el Archivo Nacional de Costa Rica, especialmente en los fondos Congreso, Gobernación, Municipal y Policía, donde se ubican numerosos documentos que mencionan la presencia de las langostas para el período 1877-1878, que muchas veces aparecen designadas como chapulines y saltones, términos que ya fueron explicados con anterioridad y donde se describen los impactos en las distintas localidades en las que se reportó la presencia de dichos insectos.

Dado el carácter descriptivo de la información, se realizó un estudio cualitativo, por lo que se hizo una selección de documentos donde aparecen datos que permiten reconstruir el impacto de las nubes de langostas en territorio costarricense durante el período de estudio, especialmente las que mencionan daños en los campos de cultivo y en los pastizales, así como las acciones emprendidas para combatir la plaga. De esas fuentes primarias se extrajeron los nombres de las localidades afectadas por la presencia de los insectos (con base en ellas se confeccionó un mapa donde se muestra la extensión de la plaga a nivel nacional) y se transcribieron las descripciones de su impacto, las cuales fueron organizadas cronológicamente y sintetizadas para su respectiva clasificación según la región del país de donde proceden los reportes.

En cuanto al aspecto geofísico, se tomó como base el análisis de las figuras elaboradas por Díaz y Alfaro (2016), a partir de los datos disponibles que atestiguan la presencia del Mega-Niño de ese mismo período que afectó a los países centroamericanos. Siguiendo lo propuesto por Díaz y Alfaro (2016), se calcularon las anomalías estandarizadas de la precipitación para la primera componente principal en el dominio $6,7-14,3^{\circ} \mathrm{N} \& 75-93,4^{\circ} \mathrm{W}$ y las anomalías de la cortante vertical del viento zonal entre los niveles de 1000 y $925 \mathrm{hPa}$, para la región central de la corriente en chorro de bajo nivel en el Caribe $\left(12,5-17,5^{\circ} \mathrm{N} \& 75-80^{\circ} \mathrm{W}\right.$, Amador, 2008; Amador, Alfaro, Rivera, \& Calderón, 2010) una zona caracterizada por vientos fuertes del este en bajo nivel. Estas dos variables se obtuvieron de la base de datos generada por Compo et al. (2011).

Ambas anomalías fueron calculadas de acuerdo al periodo base de 1871-1901, es decir treinta y un años. Por último, se obtuvieron las anomalías estandarizadas de la Temperatura Superficial del Mar (TSM) observadas entre 1876 y 1879, para el dominio comprendido desde los $6^{\circ} \mathrm{N}, 92^{\circ} \mathrm{W}$ hasta la costa pacífica centroamericana, utilizando los datos de Smith, Reynolds, Peterson y Lawrimore (2008) Dichas anomalías fueron calculadas de acuerdo al periodo base de 1862-1892 (treinta y un años). Las anomalías estandarizadas se calcularon restándole al valor mensual observado el valor medio y dividiéndolo entre la desviación estándar.

Ética, conflicto de intereses y declaración de financiamiento: los autores declaran haber cumplido con todos los requisitos éticos y legales pertinentes, tanto durante el estudio como en el manuscrito; que no hay conflictos de interés de ningún tipo, y que todas las fuentes financieras se detallan plena y claramente en la sección de agradecimientos. Asimismo, están de acuerdo con la versión editada final del documento. El respectivo documento legal firmado se encuentra en los archivos de la revista. 


\section{RESULTADOS}

La Costa Rica de la década de 1870 era un país que basaba su economía principalmente en las exportaciones del café, cultivado en el Valle Central, donde residía la mayor parte de la población costarricense que, según el censo de 1875, era de 156634 habitantes (Thiel, 2011). En cuanto a la infraestructura, lo más destacado fue el proyecto de construcción del Ferrocarril al Atlántico que para ese momento ya unía las principales ciudades del Valle Central (Alajuela, Cartago, Heredia y San José) mientras que a partir del puerto caribeño de Limón comenzaba a tenderse el otro extremo de la vía férrea que terminaría uniendo el interior del país con la costa del Caribe al finalizar la década de 1880 , lo que abriría la puerta a la colonización de los valles de Turrialba y del río Reventazón, las inversiones del capital estadounidense y el cultivo del banano a escala comercial en la antigua Comarca de Limón (Molina, 2005).

A nivel político, en 1877 se dio el retorno al poder del General Tomás Guardia Gutiérrez (1831-1882), tras los breves gobiernos de Aniceto Esquivel (1876) y Vicente
Herrera (1876-1877). Guardia gobernó dictatorialmente hasta su muerte y bajo su último mandato, prosiguió con el proceso de reforma de la institución militar, el alejamiento del control del Estado por parte de la oligarquía cafetalera y una mayor estabilidad política (Díaz, 2005).

En el aspecto ambiental, al igual que los demás países centroamericanos, Costa Rica experimentó en el siglo XIX la presencia de nubes de langostas a través de su territorio, lo que trajo consigo daños en las tierras cultivadas, especialmente para un país cuya economía se basaba en un modelo agroexportador y por tanto, susceptible a las fluctuaciones de la economía mundial. Amador y Muñoz (2015) sostienen que la plaga de langostas que afectó al territorio costarricense en el bienio 1877-1878, se centró en las poblaciones de Nicoya y Cartago. No obstante, las fuentes documentales del período evidencian que dicha plaga se expandió a lo largo y ancho del territorio costarricense, debido a los reportes procedentes de localidades ubicadas tanto en el Valle Central y en las zonas costeras y periféricas del país (Fig. 2).

Díaz y Alfaro (2016) observaron que existe una mayor incidencia de la plaga de langostas en la vertiente del

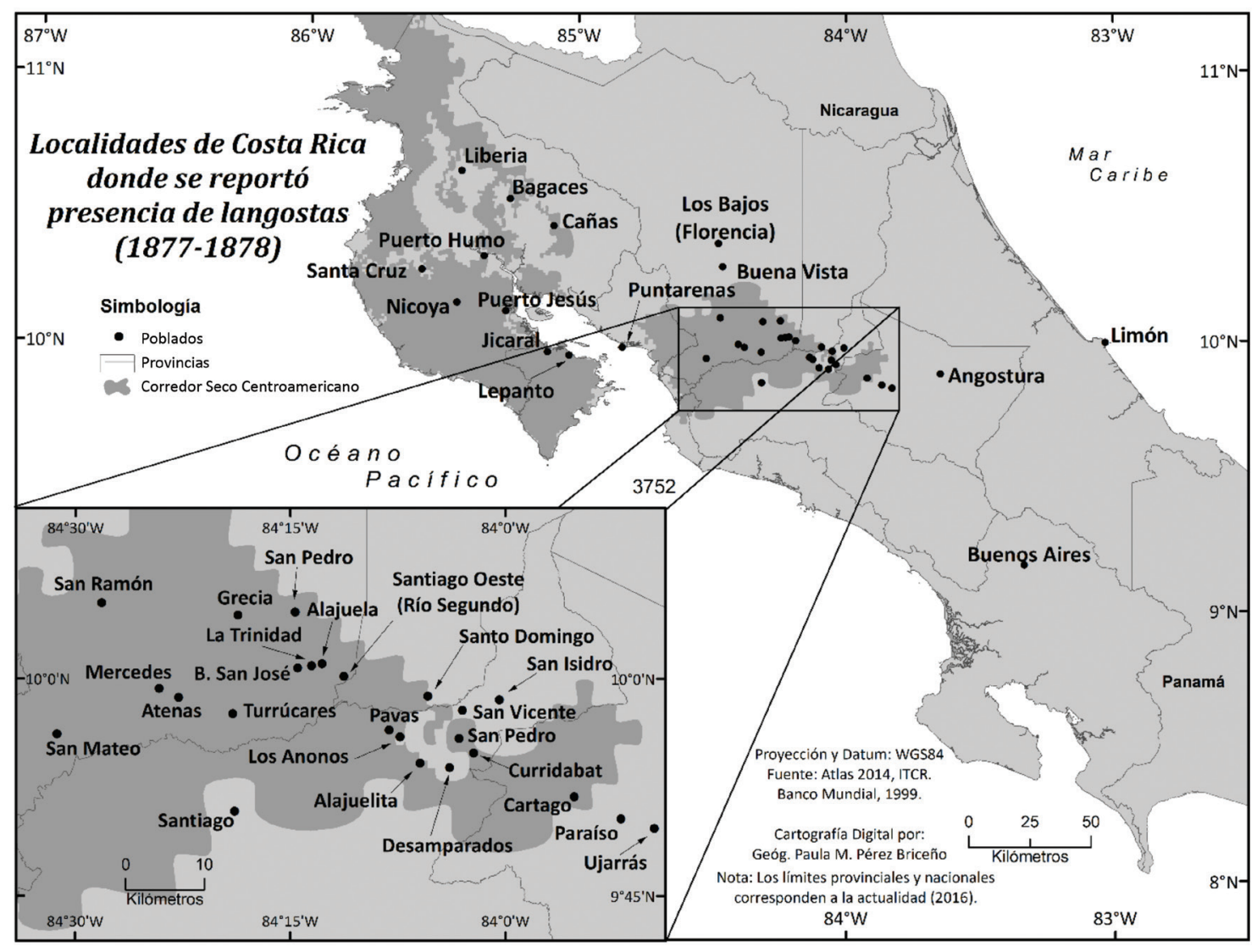

Fig. 2. Localidades de Costa Rica donde se reportó presencia de langostas (1877-1878) según los documentos consultados en el Archivo Nacional de Costa Rica (Díaz \& Alfaro, 2016). 
Pacífico más que en la del Caribe. De hecho, en la Fig. 2, en gris oscuro se demarca el Corredor Seco Centroamericano (CSC), término que designa las tierras ubicadas principalmente en la vertiente del Pacífico en América Central (entre el estado mexicano de Chiapas y Costa Rica) que son más propensas a sufrir sequías y la disminución de las precipitaciones -en el orden de los 500 y $2000 \mathrm{~mm}$ anuales- y que en el caso costarricense comprenden la provincia de Guanacaste, el norte de Puntarenas, gran parte del occidente y algunas zonas del oriente del Valle Central (Retana et al., 2012; Calvo-Solano, Quesada-Hernández, Hidalgo, \& Gotlieb, 2018). En esa época, las condiciones de sequía hicieron favorable la transición hacia la fase gregaria de las langostas, por lo que hubo una mayor cantidad de reportes en dicho espacio geográfico.

Hay que tener en cuenta que en esa época la mayor parte de la población se ubicaba en la vertiente pacífica, especialmente en el sector occidental del Valle Central, además que dicha plaga penetró a través de la costa occidental, como se desprende del análisis de los primeros reportes procedentes de la provincia de Guanacaste, fechados en 1876. Por ejemplo, en la localidad de Nicoya, en junio de ese año, el maíz (alimento de primera necesidad) escaseó y al incrementarse significativamente su precio (la fuente no especifica el monto), se hizo inalcanzable para los habitantes pobres de la localidad, afectó la asistencia de los niños a la escuela y se cerró hasta que pasara la carestía generada por la escasez de alimentos de primera necesidad (ANCR, Gobernación 28 394, 1876, f. $76 v ; 38883,1876$, ff. 36v-37). En el resto de la provincia de Guanacaste se dictaban medidas contra esta plaga de insectos (ANCR, Gobernación, 29 773, 1877, ff. 5-5v) y, en algunos lugares de esa provincia como Bagaces, se presentaban problemas para el combate de la misma (ANCR, Gobernación, 28 909, 1877, ff. 2,5 y 6).

En el caso de la vecina Comarca de Puntarenas el chapulín destruyó casi todas las sementeras (ANCR, Gobernación, 4 558, 1877, f. 19). Otros puntos afectados fueron Jicaral y Lepanto, en la Península de Nicoya (ANCR, Gobernación, 4 558, 1877, f. 18v). En esta comarca se emitieron a su vez tres informes, el primero referente al estado de la plaga de la langosta en dicho territorio, el segundo acerca de la cantidad recaudada para el combate de los insectos y el tercero sobre el impacto de las langostas en el territorio puntarenense (en particular para la agricultura local) y las medidas ejecutadas para combatirla (ANCR, Gobernación, 4 558, 1877, ff. 21-21v). Para finales de 1877 se dio la reaparición de la langosta en Esparza y otros puntos de la comarca puntarenense (ANCR, Gobernación, 4 558, 1877, snf).
Al transcurrir el tiempo, los insectos se fueron multiplicando rápidamente, por lo que millones de langostas expandieron su radio de acción a través del territorio costarricense, dejando tras de sí tierras cultivadas sumamente afectadas, por lo que su aglomeración generó temor en los ciudadanos de Costa Rica entre 1877 y 1878. Este es el caso de la provincia de Cartago en donde las fuentes consignaron que la vertiginosa reproducción del saltón provocaría una presencia masiva del insecto, incluso mayor que la observada hasta el momento (ANCR, Municipal Cartago, 17 643, 1877, f. 394). Esas nubes de insectos también se extendieron a la villa del Paraíso, la que se reportó considerablemente afectada por la plaga de langostas (ANCR, Municipal Cartago, 1877, 17 643, f. 384).

Para 1877, no solamente la provincia de Cartago reportó la plaga de langostas. Se indicó la presencia de chapulines en las Ilanuras del norte del país, propiamente en San Carlos, donde se mantuvo con fuerza en la zona de Los Bajos, hoy día Florencia, donde inclusive se emplearon la pólvora y los rifles para combatirla (ANCR, Gobernación, 28 162, 1877, f. 1; 28 100, 1877, ff. 85 y 113). En el caso de la Comarca de Limón, se presentaron algunas nubes de langostas, pero a diferencia de otras zonas del país, como ya se señaló, el impacto fue mínimo (ANCR, Gobernación, 39 227, 1877, ff. 91-93).

Mientras tanto, la menor incidencia en la vertiente del Caribe puede estar ligada a condiciones climáticas de mayor humedad y a la escasa población que la habitaba, en especial en la Comarca de Limón, que de acuerdo con el censo de 1875 contaba con 3064 habitantes y en ese momento experimentaba un paulatino proceso de colonización por la construcción del Ferrocarril al Atlántico que se consolidaría al finalizar el siglo pasado con el desarrollo de la actividad bananera (Viales, 2001; Thiel, 2011).

El Estado costarricense debió realizar ingentes esfuerzos para combatir la plaga y para ello aportó sumas de dinero destinadas al mantenimiento de quienes asumieron la labor de destruir las nubes de langostas. En ese sentido, se le retribuyó al agricultor Demetrio Tinoco Iglesias (1847-1902) la suma de 98 pesos equivalentes al valor de dos novillos que ofreció para dicho fin (ANCR, Gobernación, 25 135, 1878, f. 1). En julio de 1877 se gestionaron 1000 pesos para los trabajadores empeñados en el control de la langosta, suma que fue complementada por otros 100 pesos recaudados en San José con la misma finalidad y se invirtieron 327,75 pesos para la compra de maíz elaborado que sirviera de alimento para quienes combatían la langosta en los barrios capitalinos de San Vicente y San Isidro (ANCR, Gobernación, 27 038, 1877, ff. 7-7v; 28 100, 1877, f. 117). 
Otro ejemplo acerca del impacto a nivel económico que tuvo la presencia de las langostas en la población de la Costa Rica de 1877, fue el gasto que se hizo para el control de la misma, tal es el caso de los 2000 pesos que se recolectaron para las personas dedicadas a la lucha contra las langostas, según lo constató el jefe político de San Mateo, en la provincia de Alajuela (ANCR, Gobernación, 4 559,1877, f. 13). De modo similar, se emitió una lista sobre la cantidad adeudada respecto a la manutención de los trabajadores dedicados a la destrucción de la langosta, así como una cuenta de gastos hechos para eliminar dicha plaga en San Carlos, en donde se hicieron al menos dos pagos de 78,50 pesos para el sostenimiento de los trabajadores (ANCR, Gobernación, 4 559, 1877, ff. 13v, $14 v, 15$ y 20v; 7348,1878 , snf). Esta condición se mantuvo inclusive hasta el año de 1878.

En junio de 1878, el gobierno dio una orden a la gobernación de la Comarca de Limón para fomentar el cultivo de raíces (yuca, papa y ñame) como medida preventiva ante la posible escasez de cereales por la plaga de langostas (ANCR, Gobernación, 2444,1878 , snf). También se emitió un acuerdo relativo a la destrucción del chapulín en su etapa de saltón porque se encontraba bastante extendido por diversos puntos del territorio costarricense (ANCR, Congreso, 21 108, 1878, f. 2). Además de los campos de cultivo de cereales, el ganado se vio perjudicado por el paso de las nubes de langostas debido a la destrucción que causaban en los pastizales, como ocurrió en Buena Vista de San Carlos, por lo que hubo que atender los problemas generados con la alimentación de los semovientes (Peraldo et al., 2011).

Ese mismo año, varias poblaciones próximas a la capital reportaron la presencia del insecto, entre ellas las de Curridabat, Desamparados, Los Anonos (Escazú), Mojón (hoy día Montes de Oca) y Pavas, cuya población se dedicaba a las labores agrícolas y cuyos habitantes debieron hacer frente a la emergencia suscitada por las langostas mediante la eliminación de los enjambres (ANCR, Gobernación, 38 979, 1878, ff. 8, 32, 45 y 48). Debido al costo que implicaba el combate del chapulín, los vecinos de Alajuelita, otra localidad próxima a la capital, acordaron según las posibilidades económicas de cada uno de los residentes, cuyas tierras agrícolas estaban siendo afectadas por la plaga (ANCR, Policía, 11 767, 1878, ff. 2-3).

Además, se dio la colaboración del personal militar en las labores de combate contra las nubes de langostas, ya que la plaga coincide con el retorno al poder del General Guardia y el ejército costarricense movilizó algunos recursos para erradicarla (ANCR, Gobernación, 36 407, 1877, snf y 7301,1878 , snf). Incluso el obispo Luis Bruschetti (1826-1881), quien se desempeñaba como
Administrador Apostólico de la Diócesis de San José de Costa Rica, promulgó una carta en la que motivaba a la feligresía costarricense a elevar plegarias al cielo para detener el avance de la plaga (Sanabria, 1973). Según un testimonio de la época, las nubes de insectos eran tan densas que dificultaba distinguir a otras personas ubicadas a una distancia corta (Peraldo et al., 2011).

Es factible que al variar las condiciones meteorológicas que dieron lugar al Mega-Niño, la fase de gregarización se debilitara y las nubes de langostas se desplazaran hacia otras zonas. Algunos reportes de la época mencionan el traslado de los insectos hacia la costa del Pacífico (ANCR, Gobernación, 27 554, 1878, f. 7) e incluso hacia el sur del país, donde se reportó su presencia en Buenos Aires (ANCR. Gobernación, 26 926, 1877, ff. 1-10), un asentamiento surgido como resultado de la expansión de la frontera agrícola que tenía lugar en la cuenca del río Grande de Térraba (Díaz, 2015). De acuerdo con Peraldo et al. (2011), la plaga de langostas se desplazó hacia la vecina Colombia, donde causó cuantiosos daños en el Valle del Cauca, ya que la destrucción de las cosechas provocó una carestía que generalizó el hambre en esta región e hizo que el gobierno colombiano solicitara ayuda a los países vecinos para atender la emergencia.

\section{DISCUSIÓN}

De acuerdo a lo mostrado por Aceituno et al. (2009), la estación meteorológica de San José, Costa Rica -ubicada en la Oficina de Estadística- presentó una predominancia de condiciones con déficit de precipitación desde junio de 1877 hasta agosto de 1878, con respecto al valor medio observado en esa estación y congruente con la presencia de un Mega-Niño en el Pacífico ecuatorial del este. Para complementar este resultado, Díaz y Alfaro (2016) utilizaron los datos de lluvia de la base de Compo et al. (2011) para el dominio en rejilla de 6,7-14,3N \& 75-93,4W, que abarca la mayor parte del istmo centroamericano y observaron un período de condiciones secas dominantes en América Central desde agosto de 1876 hasta abril de 1878 (Fig. 3), debido a que se presentó una predominancia de anomalías negativas durante el periodo antes descrito. Según Díaz y Alfaro (2016), los datos colectados por Maison (1879) y observados en la estación de San José, Costa Rica, mostraron también condiciones deficitarias de precipitación durante el período 1873-1877, al compararlo con el registro 1866-1878.

Según Díaz y Alfaro (2016), el resultado anterior, podría estar relacionado con el aumento de la magnitud del viento zonal (componente del viento paralela a los paralelos geográficos) en la región del Caribe, relacionado con 


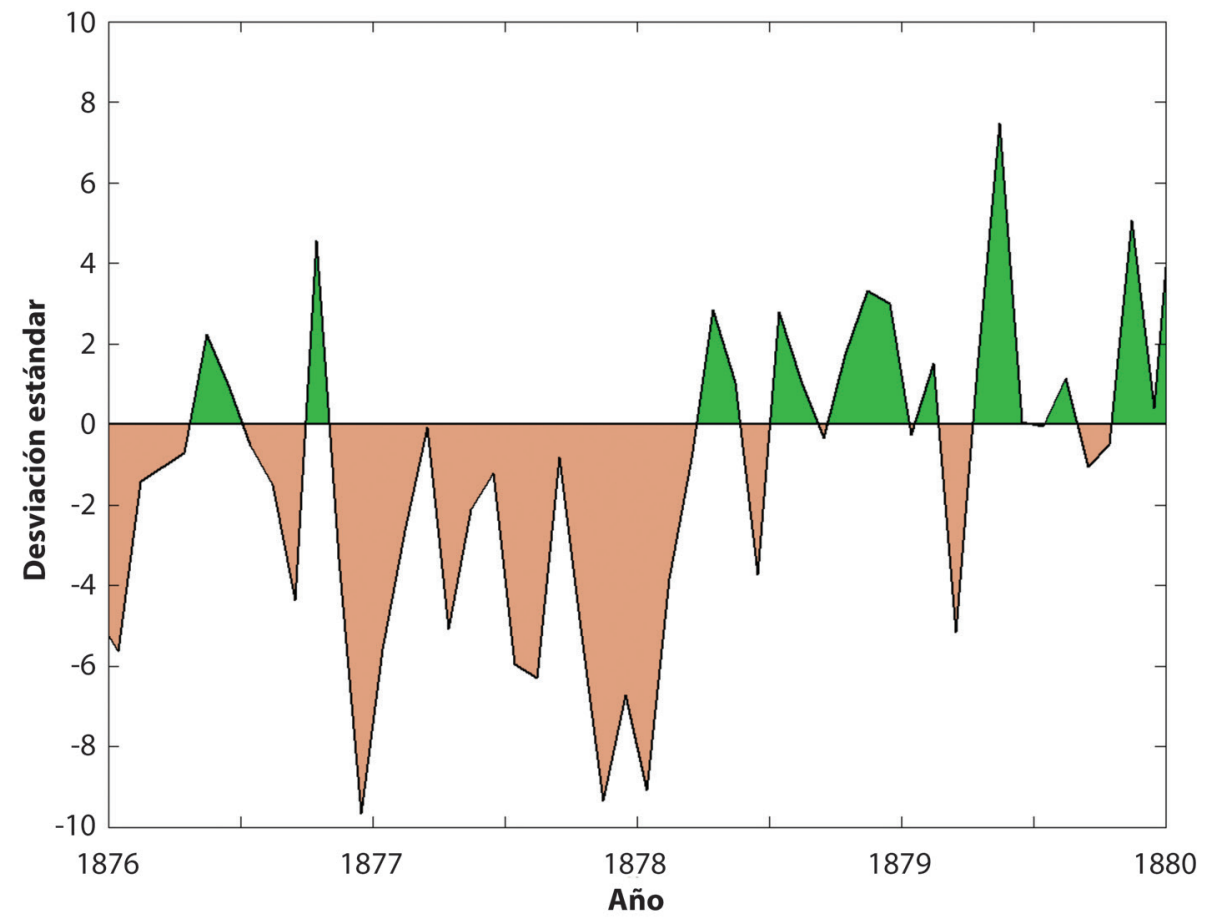

Fig. 3. Anomalías estandarizadas de la precipitación para la primera componente principal en el dominio 6,7-14,3N \& 75-93,4W, de acuerdo a los datos de Compo et al. (2011). Anomalías calculadas de acuerdo al periodo base de 1871-1901 (Basada y modificada de Díaz \& Alfaro, 2016).

valores de fuerte cortante vertical del viento zonal (o sea una marcada diferencia en magnitud y dirección, entre el viento en niveles bajos y altos de la troposfera), observados entre junio de 1876 y noviembre de 1878 que no favorecen la formación de sistemas mesoescalares sobre el istmo centroamericano, especialmente sobre la vertiente del Pacífico (Fig. 4) y que son responsables de la formación de sistemas convectivos, asociados principalmente a la formación de tormentas (Alfaro, 2007a,2007b).

En una recopilación de datos de temperatura superficial del aire para la estación de San José, Costa Rica, hecha por Solano, Díaz y Amador (2013), se observó que el año de 1878 presentó anomalías positivas. En esta recopilación no se pudieron recolectar datos de los años 1876 y 1877. Debido a lo anterior, Díaz y Alfaro (2016) utilizaron datos de temperatura superficial de mar (TSM) para el Océano Pacífico de la base de Smith et al. (2008). El dominio utilizado comprendió desde los $6 \mathrm{~N}$ y $92 \mathrm{~W}$ hasta la costa pacíica centroamericana. Al analizar las anomalías normalizadas de la TSM observada entre 1876 y 1879, Díaz y Alfaro (2016) notaron condiciones cálidas dominantes desde octubre de 1876 hasta octubre de 1879 en las aguas del pacífico centroamericano (Fig. 5). Los datos recolectados por Maison (1879) en la estación de San José, mostraron también que durante los años
1877-1878 se observaron anomalías positivas de la temperatura superficial del aire, período que estuvo precedido por anomalías negativas durante 1870-1876 (Díaz \& Alfaro, 2016).

Retana (2000) señala que las elevaciones en la temperatura ambiental y una irregular distribución de las precipitaciones, tales como las que se experimentan en el Pacífico Norte de Costa Rica durante años influenciados por El Niño pueden catalizar períodos de cópula y ovipostura de la langosta, así como contribuir al hacinamiento, que es el detonante para la gregarización. Obsérvese que los resultados de este autor corresponden al Pacífico Norte de Costa Rica y que no necesariamente son directamente aplicables al resto del Pacífico centroamericano, lo cual requeriría un estudio posterior.

No obstante, a partir de la información meteorológica existente que atestiguan la presencia del Mega-Niño en Costa Rica durante el período 1877-1878 y de acuerdo con lo apuntado por Retana (2000), los enjambres de langostas experimentaron condiciones propicias para alcanzar el proceso de gregarización y expandirse por amplias zonas del territorio costarricense, especialmente las más habitadas y donde había un mayor desarrollo de la agricultura y la ganadería en esa época, según se 


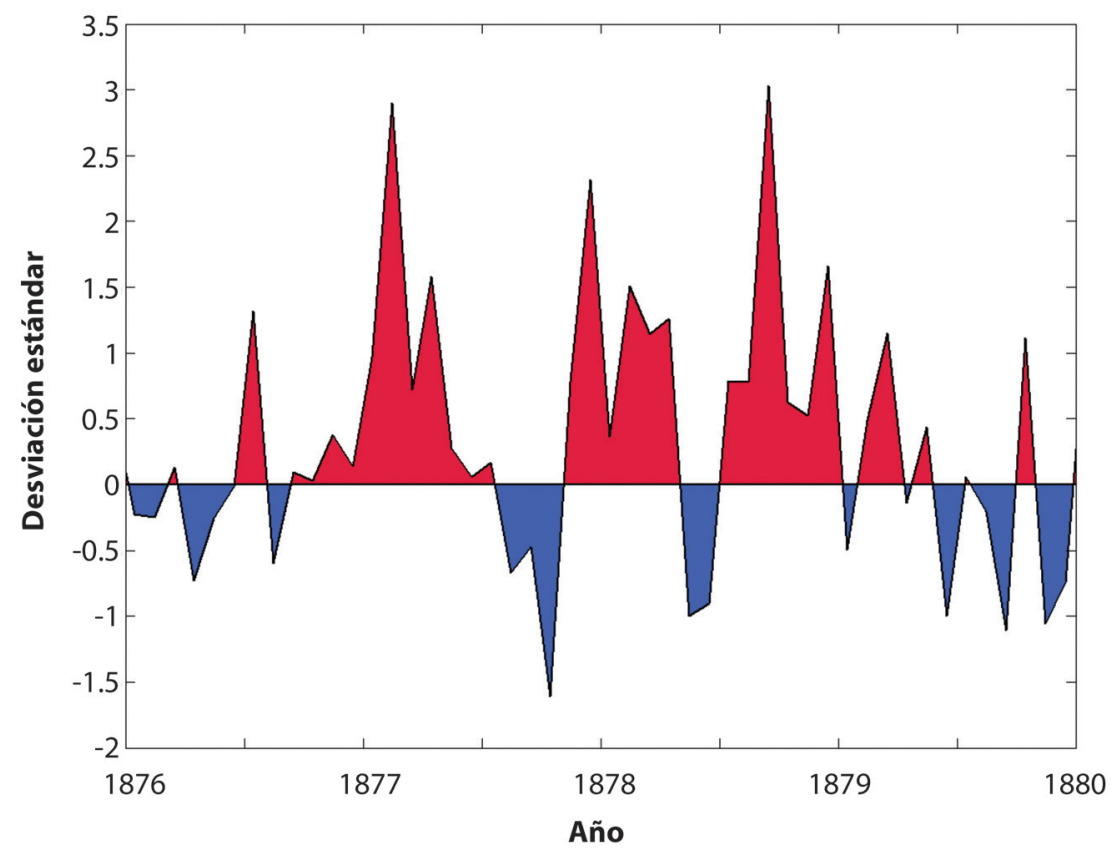

Fig. 4. Anomalías estandarizadas de la cortante vertical del viento zonal entre los niveles de 1000 y $925 \mathrm{hPa}$, para la región central de vientos fuertes de la corriente en chorro de bajo nivel en el Caribe (12,5-17,5N \& 75-80W, Amador 2008; Amador et al., 2010), utilizando los datos de Compo et al. (2011). Anomalías calculadas de acuerdo al periodo base de 1871-1901 (Basada y modificada de Díaz \& Alfaro, 2016).

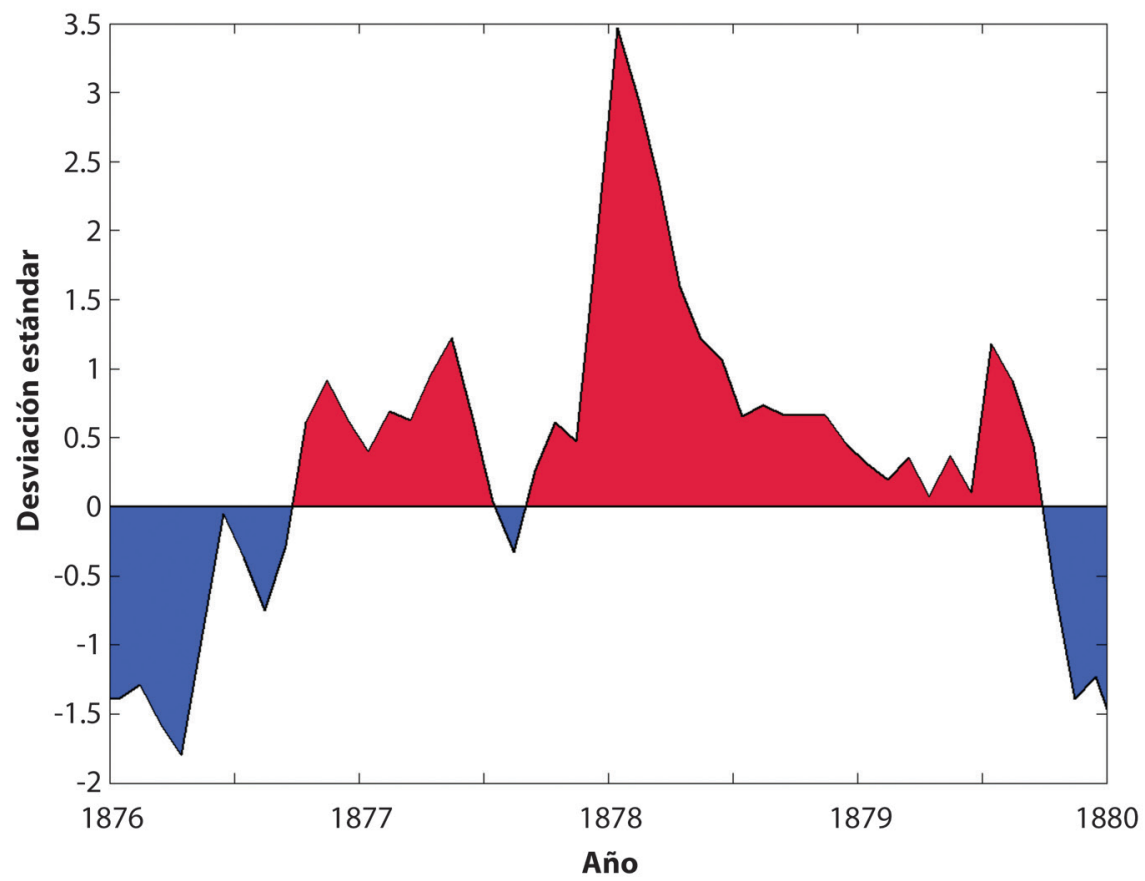

Fig. 5. Anomalías estandarizadas de la TSM observada entre 1876 y 1879 , para el dominio comprendido desde los $6 \mathrm{~N}$ y $92 \mathrm{~W}$ hasta la costa pacífica centroamericana, utilizando los datos de Smith et al. (2008). Anomalías calculadas de acuerdo al periodo base de 1862-1892 (Basada y modificada de Díaz \& Alfaro, 2016). 
desprende de la documentación conservada en los acervos del Archivo Nacional de Costa Rica.

La presente investigación concluye que durante el evento del Mega-Niño de 1877-1878, se dieron condiciones que favorecieron el impacto de una plaga de langostas en amplias zonas del territorio costarricense de acuerdo con las fuentes documentales producidas durante el período de estudio. No obstante, la relación existente entre los fenómenos de variabilidad climática como El Niño, con respecto al desarrollo de las plagas de langostas, sigue siendo bastante compleja y a la fecha no es entendida en su totalidad. En este sentido, Amador y Muñoz (2015) señalan que el análisis de la evidencia disponible no es concluyente y por tanto no se ha podido constatar una relación directa entre ambos procesos.

Sin embargo, Retana (2000) sostiene que hay un $72 \%$ de coincidencia entre los años en que se presenta el fenómeno de El Niño y los ataques masivos de langostas en la zona baja de la Región Chorotega (que corresponde a la provincia de Guanacaste, Costa Rica), por lo que sugiere que algunas de las alteraciones climáticas causadas por dicho fenómeno océano-atmosférico, en particular las afectaciones en la temperatura ambiental y los patrones de precipitaciones, podrían favorecer la gregarización de estos insectos. Según Fernández y Ramírez (1991) y Chacón (1993), los eventos de El Niño provocan "una estación lluviosa irregular y un período seco prolongado" sobre la vertiente pacífica costarricense.

De acuerdo con Díaz y Alfaro (2016), el periodo 18771878 se caracterizó por el predominio de condiciones más cálidas, secas y ventosas en el istmo centroamericano. Tales condiciones concuerdan con las señaladas por Fernández y Ramírez (1991), Chacón (1993) y Amador, Alfaro, Lizano y Magaña (2006) como las normales en la vertiente pacífica de América Central durante un evento de El Niño en el Pacífico ecuatorial oriental. El escenario climático descrito con anterioridad corresponde al Mega-Niño analizado por Aceituno et al. (2009) para el bienio 1877-1878, por lo que no se favorece la formación de sistemas mesoescalares de convección profunda en la vertiente pacífica y también de los huracanes en la cuenca del Atlántico Norte. Lo anterior concuerda también con las condiciones secas registradas en dicho período (Alfaro, 2007a, 2007b) y que según Retana (2000), son propicias para la aparición de una plaga de langostas en la vertiente del Pacífico en Costa Rica.

Los datos de las fuentes documentales consultadas constituyen una evidencia del impacto del paso de las nubes de langostas por el territorio costarricense durante el período de estudio ya que los reportes procedentes tanto de las zonas más habitadas como de las de mayor desarrollo agropecuario en ese momento. Aunque no se hicieron estimaciones de los daños provocados por esta plaga, es factible que pudieron ascender a varios miles de pesos de la época porque las nubes de langostas se esparcieron a través de Costa Rica, incluso en áreas donde la frontera agrícola estaba en proceso de expansión como la Comarca de Limón, las llanuras del Norte y la cuenca del Río Grande de Térraba. Aun así, algunas fuentes evidenciaron algunos montos que se destinaron para el combate de la langosta que oscilaron entre los 78,50 y los 2000 pesos (en esa época el precio de un novillo podía costar unos 49 pesos).

La mayoría de los reportes de langostas analizados proceden de poblaciones ubicadas en la vertiente del Pacífico, no solo de la provincia de Guanacaste y de la comarca de Puntarenas, sino también del Valle Central, precisamente en las áreas comprendidas dentro del CSC. Debido a lo anterior, puede concluirse que esa zona fue un espacio propicio para el desarrollo de las plagas de langostas, en particular durante los eventos de El Niño y los Mega-Niños, que generaron condiciones climáticas predominantemente secas e hicieron posible la difusión de las nubes de insectos a otras zonas del país.

Las mismas fuentes atestiguan los daños provocados por las nubes de insectos a su paso por poblados y tierras cultivadas, por lo que la población debió enfrentar la presencia de millones de langostas y combatirla mediante el empleo de pólvora o armas de fuego para tratar de disgregarlas y estimulando la agricultura para el consumo interno en las zonas menos afectadas por la plaga. Sin embargo, las condiciones meteorológicas propias de un fenómeno como el Mega-Niño debieron dificultar dicha labor, especialmente en las zonas ubicadas dentro del CSC que son propensas a sufrir el embate de las sequías.

Es necesario hacer más investigaciones que permitan esclarecer aún más la forma en que las condiciones meteorológicas asociadas a fenómenos como El Niño - y especialmente en períodos donde se presentan los MegaNiños que se caracterizan por sus grandes impactos a escala planetaria - favorecen el desarrollo de las nubes de langostas y otras plagas que provocan graves impactos en las actividades agropecuarias de países como los de América Central, cuyas economías históricamente se han basado en la producción agrícola, tanto para sus exportaciones como para abastecer sus mercados internos.

\section{AGRADECIMIENTOS}

La presente investigación se elaboró en el marco del Proyecto El Mega-Niño de 1876-1878 y su impacto social en América Central (805-B4-228), adscrito al Programa 
de Estudios Sociales de la Ciencia, la Técnica y el Medio Ambiente (PESCTMA, 805-A4-906) del Centro de Investigaciones Geofísicas (CIGEFI) de la Universidad de Costa Rica. También a las iniciativas 805-B7-286 (Espacio de Estudios Avanzados de la Universidad de Costa Rica, UCREA), B6-143, B7-507 \& B8-766 (Redes)(UCR y Consejo Nacional para Investigaciones Científicas y Tecnológicas, Ministerio de Ciencia, Tecnología y Telecomunicaciones o CONICIT-MICITT), 808-B5-298. Un avance de la misma se presentó en la Mesa de Historia Ambiental en el marco del XIII Congreso Centroamericano de Historia que se celebró en Tegucigalpa (Honduras), en julio de 2016. Los autores agradecen a Paula Marcela Pérez Briceño por su colaboración en la confección del mapa que ubicó los puntos afectados por la plaga de langostas en Costa Rica entre 1876 y 1878 y a Óscar Calvo y Luis Quesada por su aporte en la búsqueda de fuentes bibliográficas.

\section{REFERENCIAS}

Aceituno, P., Prieto, M.R., Solari, M.E., Martínez, A., Poveda, G., \& Falvey, M. (2009). The 1877-1878 El Niño episode: associated impacts in South America. Climatic Change, 92(34), 389-416. DOI: 10.1007/s10584-008-9470-5

Alfaro, E., \& Amador, J.A. (1996). El Niño-Oscilación del Sur y algunas series de temperatura máxima y brillo solar en Costa Rica. Tópicos Meteorológicos y Oceanográficos, 3(1), 19- 26.

Alfaro, E. (2007a). Escenarios climáticos para temporadas con alto y bajo número de huracanes en el Atlántico. Revista de Climatología, 7, 1-13.

Alfaro, E. (2007b). Uso del análisis de correlación canónica para la predicción de la precipitación pluvial en Centroamérica. Revista Ingeniería y Competitividad, 9(2), 33-48.

Amador, J.A., Alfaro, E., Lizano, O., \& Magaña, V. (2006). Atmospheric forcing in the Eastern Tropical Pacific: A review. Progress in Oceanography, 69, 101-142. DOI: 10.1016/j.pocean.2006.03.007

Amador, J.A., Alfaro, E., Rivera, E., \& Calderón, B. (2010). Climatic Features and Their Relationship with Tropical Cyclones Over the Intra-Americas Seas. In J. Elsner, R. Hodges, J. Malmstadt, \& K. Scheitlin (Eds.), Hurricanes and Climate Change (pp. 149-173). New York, U.S.A.: Springer. DOI: 10.1007/978-90-481-9510-7_9

Amador, J.A., \& Muñoz, G. (2015). Moduladores climáticos de baja frecuencia y las plagas de langostas en Mesoamérica. En G. Peraldo (Ed.), Plagas de langostas en América Latina. Una perspectiva multidisciplinaria (pp. 57-97). San José, Costa Rica: Editorial Nuevas Perspectivas.

Amador, J.A. (2008). The Intra-Americas Seas Low-Level Jet (IALLJ): Overview and Future Research. Annals of the New York Academy of Sciences, 1146(1), 153-188. DOI: 10.1196/annals.1446.012
Calvo-Solano, O.D., Quesada-Hernández, L., Hidalgo, H., \& Gotlieb, Y. (2018). Impacto de las sequías en el sector agropecuario del Corredor Seco Centroamericano. Agronomía Mesoamericana, 29(3), 695-709. DOI:10.15517/ma.v29i3.30828.

Chacón, R. (1993). Aspectos de la influencia del fenómeno de El Niño en el clima costarricense. Revista Geográfica de América Central, 27, 53-66.

Compo, G.P., Whitaker, J.S., Sardeshmukh, P.D., Matsui, N., Allan, R.J., Yin, X., ... Worley, S.J. (2011). The Twentieth Century Reanalysis Project. Q.J.R. Meteorological Society, 137, 1-28. DOI: $10.1002 /$ qj.776

Díaz, D. (2005). Construcción de un Estado moderno. Política, Estado e identidad nacional en Costa Rica, 1821-1914. San José, Costa Rica: Editorial de la Universidad de Costa Rica.

Díaz, R., \& Alfaro, E. (2016). El Mega-Niño de 1877-1878 y su impacto social en Costa Rica. Tópicos Meteorológicos y Oceanográficos, 15(2), 20-32.

Díaz, R. (2015). La transformación de las sabanas de Buenos Aires de Puntarenas, Costa Rica (1870-2010). En C. Lértora (Comp.), Sustentabilidad y conservación: EcoEpisteme (pp. 109-137). Buenos Aires, Argentina: FEPAI.

Fernández, W., \& Ramírez, P. (1991). El Niño, La Oscilación del Sur y Sus Efectos en Costa Rica: Una Revisión. Tecnología en Marcha, 11(1), 3-10.

Flores, F. (2015). Las plagas de langosta en el área maya prehispánica. En G. Peraldo (Ed.), Plagas de langostas en América Latina. Una perspectiva multidisciplinaria (pp. 29-56). San José, Costa Rica: Editorial Nuevas Perspectivas.

Maison, F. (1879). Observaciones meteorológicas verificadas en la ciudad de San José. San José, Costa Rica: Imprenta Nacional.

Ministerio de Agricultura y Ganadería. (2015). Guía Técnica. Para el muestreo de Langosta voladora. Costa Rica. Recuperado de https://app.sfe.go.cr/intranet/documentos/planes_ de_accion/Plan_de_Accion_Langosta.pdf

Molina, I. (2005). Del legado colonial al modelo agroexportador. Costa Rica (1821-1914). San José, Costa Rica: Editorial de la Universidad de Costa Rica.

Mörner, N.A. (1993). Present El Niño - Enso events and past super-Enso events. Bulletin d'Institut français d'études andines, 22(1), 3-12.

Payne, E. (1988). Organización productiva y explotación indígena en el Área Central de Costa Rica (1580-1700) (Tesis de Licenciatura). Universidad de Costa Rica, San José, Costa Rica.

Peraldo, G., Solano, F., \& Quesada, A. (2011). La plaga de langostas en Costa Rica entre 1850 y 1950. En C. Lértora (Coord.), Geonaturalia. Geografía e Historia Natural: hacia una historia comparada. Estudio a través de Argentina, México, Costa Rica y Paraguay (pp. 139-184). Buenos Aires, Argentina: Ediciones FEPAl. 
Retana, J., Alvarado, L., Araya, C., Sanabria, N., Solano, J., Solera, M., \& Alfaro, M. (2012). Caracterización del corredor seco en Costa Rica. Tópicos Meteorológicos y Oceanográficos, 11(1), 18-29.

Retana, J.A. (2000). Relación entre algunos aspectos climatológicos y el desarrollo de la langosta centroamericana Schistocerca piceifrons piceifrons en el Pacífico Norte de Costa Rica durante la fase cálida del fenómeno El Niño-Oscilacion Sur (ENOS). Tópicos Meteorológicos y Oceanográficos, 7(2), 73-87.

Sanabria, V.M. (1973). La primera vacante de la Diócesis de San José. San José, Costa Rica: Editorial Costa Rica.

SENASICA-DGSV. (2016). Ficha Técnica. Langosta Centroamericana [Schistocerca piceifrons piceifrons (Walker, 1870)] (Orthoptera:Acrididae).Tecámac, México: Servicio Nacional de Sanidad, Inocuidad y Calidad Agroalimentaria-Dirección General de Sanidad VegetalCentro Nacional de Referencia Fitosanitaria-Grupo
Especialista Fitosanitario. Recuperado de https://www. gob.mx/cms/uploads/attachment/file/157817/Ficha_t_ cnica_langosta.pdf

Smith, T.M., Reynolds, R.W., Peterson, T.C., \& Lawrimore, J. (2008). Improvements to NOAA's Historical Merged Land-Ocean Surface Temperature Analysis (1880-2006). Journal of Climate, 21, 2283-2296. DOI: 10.1175/2007JCLI2100.1

Solano, F.J., Díaz, R.E., \& Amador, J.A. (2013). La institucionalización de la meteorología en Costa Rica (1860-1910). San José, Costa Rica: Editorial Nuevas Perspectivas.

Thiel, B.A. (2011). Monografía de la población de la República de Costa Rica en el siglo XIX. Población y Salud en Mesoamérica, 9(1), 1-54.

Viales, R. (2001). La colonización agrícola de la Región Atlántica/ Caribe costarricense entre 1870 y 1930 . El peso de la política agraria liberal y de las diversas formas de apropiación territorial. Anuario de Estudios Centroamericanos, $27(2), 57-100$.

\section{APÉNDICE 1}

Fuentes legales

Archivo Nacional de Costa Rica (ANCR).

Congreso, 21108 (1878).

Gobernación 2444 (1878), 4558 (1877), 4559 (1877), 7301 (1878), 7348 (1878), 25135 (1878), 26926 (1877), 27038

(1877), 27554 (1878), 28100 (1877), 28162 (1877), 28394 (1876), 28909 (1877), 29773 (1877), 36407 (1877), 38883 (1876), 38979 (1878) y 39227 (1877).

Municipal Cartago 17643 (1877).

Policía 11767 (1878). 